\title{
The GlueX Start Counter Detector
}

\author{
E. Pooser ${ }^{\mathrm{a}, \mathrm{b}, *}$, F. Barbosa ${ }^{\mathrm{a}}$, W. Boeglin ${ }^{\mathrm{b}}$, C. Hutton ${ }^{\mathrm{a}}$, M.M. Ito ${ }^{\mathrm{a}}$, M. Kamel ${ }^{\mathrm{b}}$, P. Khetarpal ${ }^{\mathrm{b}}$, A. LLodra ${ }^{\mathrm{b}}$, \\ N. Sandoval ${ }^{\mathrm{a}}$, S. Taylor ${ }^{\mathrm{a}}$, T. Whitlatch ${ }^{\mathrm{a}}$, S. Worthington ${ }^{\mathrm{a}}$, C. Yero ${ }^{\mathrm{b}}$, B. Zihlmann $^{\mathrm{a}}$ \\ ${ }^{a}$ Thomas Jefferson National Accelerator Facility, Newport News, VA 23606, USA \\ ${ }^{b}$ Florida International University, Miami, FL, 33199, USA
}

\begin{abstract}
The design, simulation, fabrication, calibration, and performance of the GLuEX Start Counter detector is described. The Start Counter was designed to operate at integrated rates of up to $9 \mathrm{MHz}$ with a timing resolution in the range of 500 to 825 ps (FWHM). The Start Counter provides excellent solid angle coverage, a high degree of segmentation for background rejection, and can be utilized in the level 1 trigger for the experiment. It consists of a cylindrical array of 30 thin scintillators with pointed ends that bend towards the beam line at the downstream end. Magnetic field insensitive silicon photomultiplier detectors were used as the light sensors.
\end{abstract}

Keywords: GuueX, Multi-Pixel Photon Counter, Plastic Scintillator, Silicon Photomultiplier

\section{Introduction}

The GLuEX experiment, staged in Hall D at the Thomas Jefferson National Accelerator Facility (TJNAF), primarily aims to study the spectrum of photo-produced mesons with unprecedented statistics in search for gluonic degrees of freedom. The coherent bremsstrahlung technique is implemented to produce a linearly polarized photon beam that impinges on a liquid $\mathrm{H}_{2}$ target. A Start Counter detector was fabricated to properly identify the photon beam buckets and to provide accurate timing information.

\section{Design}

In this section we discuss the details of the GLuEX Start Counter design including the scintillators, support structure, light sensors and read out electronics.

\subsection{Overview}

The Start Counter detector (ST), shown in Fig. 1 surrounds a $30 \mathrm{~cm}$ long liquid $\mathrm{H}_{2}$ target while providing $\sim 90 \%$ of $4 \pi$ solid angle coverage relative to the target center. The primary purpose of the ST

\footnotetext{
* Corresponding Author

Email address: pooser@jlab.org (E. Pooser)
}

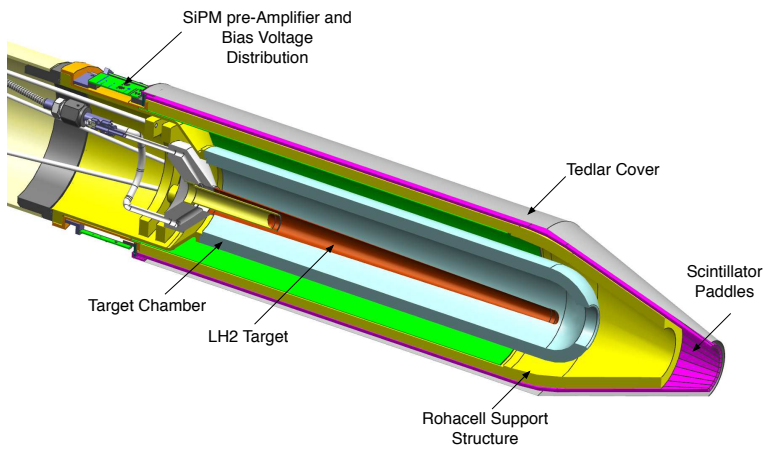

Figure 1: The GLuEX Start Counter mounted to the liquid $\mathrm{H}_{2}$ target assembly. The beam direction is oriented from left to right down the central axis of the ST.

is to identify the photon beam bucket associated with the event reaction. It is designed to operate at tagged photon beam intensities of up to $10^{8} \mathrm{\gamma} / \mathrm{s}$ in the coherent peak where the photons range in energy from 8.4 to $9.0 \mathrm{GeV}[1]$. The ST has a high degree of segmentation to limit the per paddle rates while also providing background rejection information. In order to resolve the $4 \mathrm{~ns}$ electron beam bunch structure delivered by the CEBAF to Hall-D with $6 \sigma$ accuracy, the GLuEX Start Counter time 
resolution is required to be $<350 \mathrm{ps}$. It also facilitates particle identification and can be utilized in the level 1 trigger of the GLUEX experiment during high luminosity running [2] 3 .

The ST has a cylindrical shape consisting of an array of 30 scintillators. Their pointed ends bend towards the beam line at the downstream end (Fig. 2). EJ-200 scintillator material from Eljen

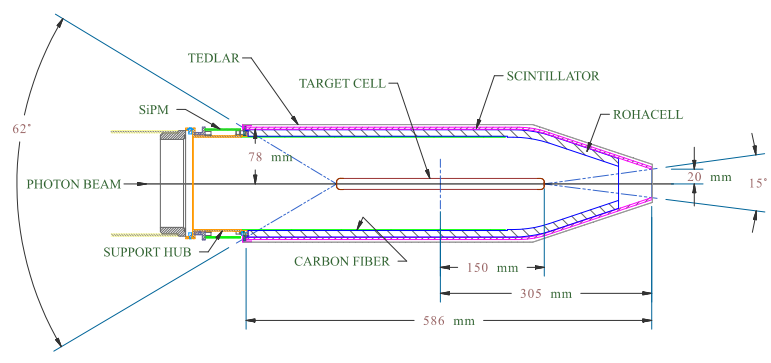

Figure 2: 2-D cross section of the Start Counter.

Technology 4 was selected for this application. EJ200 has a decay time of 2.1 ns and a long attenuation length [5]. Silicon photomultiplier (SiPM) detectors were selected as the light sensors. These sensors are not affected by the $2 \mathrm{~T}$ magnetic field produced by the GLUEX superconducting solenoid magnet. The SiPMs were placed as close as possible to the upstream end of each scintillator element thereby minimizing the loss of scintillation light [2].

\subsection{Scintillator Paddles}

Individual paddles were machined from long, thin, scintillator bars. Each paddle was manufactured to be $3 \mathrm{~mm}$ thick and diamond milled to be $600 \mathrm{~mm}$ in length and $20 \pm 2 \mathrm{~mm}$ wide. The paddles were bent around a highly polished aluminum drum by applying localized infrared heating to the bend region. The bent scintillator bars were then sent to McNeal Enterprises Inc. [6], a plastic fabrication company, where they were machined to the desired geometry illustrated in Fig. 3.

The paddles can be classified into three sections from the upstream to downstream end of the scintillator. The straight section is $39.5 \mathrm{~cm}$ in length and is oriented parallel to both the target cell and beam line. The bend region is a $18.5^{\circ}$ arc of radius $120 \mathrm{~cm}$ following the straight section. The tapered nose region is located past the target chamber and bends towards the beam line such that the tip of the nose is at a radial distance of $2 \mathrm{~cm}$ from the beam line.

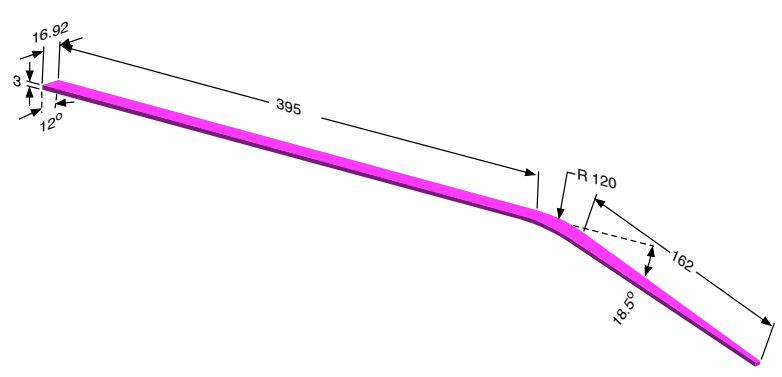

Figure 3: Start Counter single paddle geometry. Unlabeled dimensions shown are in $\mathrm{mm}$.

After the straight scintillator bar was bent to the desired geometry the two flat surfaces, oriented orthogonal to the wide top and bottom surfaces, were cut at a $6^{\circ}$ angle. During this process, the width of the top and bottom surfaces of the straight section were machined to be $16.92 \mathrm{~mm}$ and $16.29 \mathrm{~mm}$ wide respectively. Thus, each of the paddles may be rotated $12^{\circ}$ with respect to the adjacent paddles so that they form a cylindrical shape with a conical end. This geometrical design for the ST increases solid angle coverage while minimizing multiple scattering.

\subsection{Support Structure}

The ST scintillator paddles are placed atop a low density Rohacell 7 foam support structure which envelopes the target chamber, illustrated in Fig. 1 . The Rohacell is $11 \mathrm{~mm}$ thick and is rigidly attached to the support hub at the upstream end and extends along the length of paddles, partly covering the conical nose section. The cylindrical part of the Rohacell is reinforced with three layers of carbon fiber, each with a thickness of $650 \mu \mathrm{m}$; this is illustrated in green in Figures 2 and 4.

The various layers of material that comprise the ST are illustrated in Fig. 4. To ensure that the detector is light-tight, a plastic collar was placed around the top of the SiPMs at the upstream end. The collar serves as a lip to which a cylindrical sheet of light insulation film (Tedlar [8] ) is attached. The nose section is covered by a cone of Tedlar which connects to the cylindrical section. An additional cone of Tedlar is taped to the nose of the Rohacell and attached to the top Tedlar cone layer in order to ensure light-tightness. A summary of the materials utilized in the ST are presented in Table 1 . 


\begin{tabular}{lcccc}
\hline \multicolumn{1}{c}{ Item } & Brand name & Material & Thickness $(\mathbf{m m})$ & Density $\left(\mathrm{g} / \mathrm{cm}^{3}\right)$ \\
\hline Carbon fiber support & Carbon fiber & Carbon & 1.950 & 1.523 \\
Rohacell support & Rohacell & Polymethacrylimide & 11.0 & 0.075 \\
Radial shims & Kapton & Polyimide (type HN) & 0.762 & 1.42 \\
Reflective film & Aluminum foil & Aluminum & 0.016 & 2.70 \\
Scintillator & EJ-200 & Polyvinyltoluene & 3.0 & 1.023 \\
Bundling wrap & Stretch film & Polyethelene & 0.101 & 0.917 \\
Light insulation film & Tedlar & Polyvinyl Fluoride & 0.050 & 1.50 \\
\hline
\end{tabular}

Table 1: Start Counter materials.

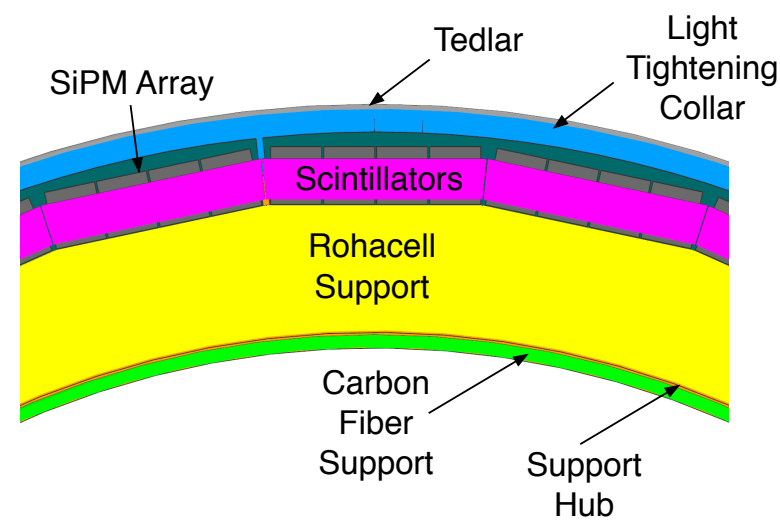

Figure 4: Start Counter materials.

\subsection{SiPM Readout Detectors}

Each scintillator bar is read out with an array of four magnetic field insensitive Hamamatsu S10931050P multi-pixel photon counters (MPPCs) 9]. Studies of several photo-detectors were performed in the initial design phase of the ST 10. Based on these studies, the S10931-050P model was selected. An individual $3 \times 3 \mathrm{~mm}^{2} \mathrm{MPPC}$, here referred to as a "SiPM", consists of 3600 individual $50 \times 50 \mu \mathrm{m}^{2}$ avalanche photo-diode (APD) pixel counters operating in Geiger mode. The signal output from each SiPM is the sum of the outputs from all 3600 APD pixels 11 .

The SiPM detectors are housed in a ceramic case that is surface mounted to a custom-fabricated printed circuit board (PCB). The PCB is rigidly attached to the lip of the upstream support hub. The individual ST scintillators are coupled to the SiPM arrays via an $250 \mu \mathrm{m}$ air gap.

\subsection{Readout Electronics}

There are three primary components of the ST detector readout system. The first component
"ST1", shown in Fig. 5 , collects scintillation light from three paddles independently and distributes the bias voltages for the SiPMs. Each array of four

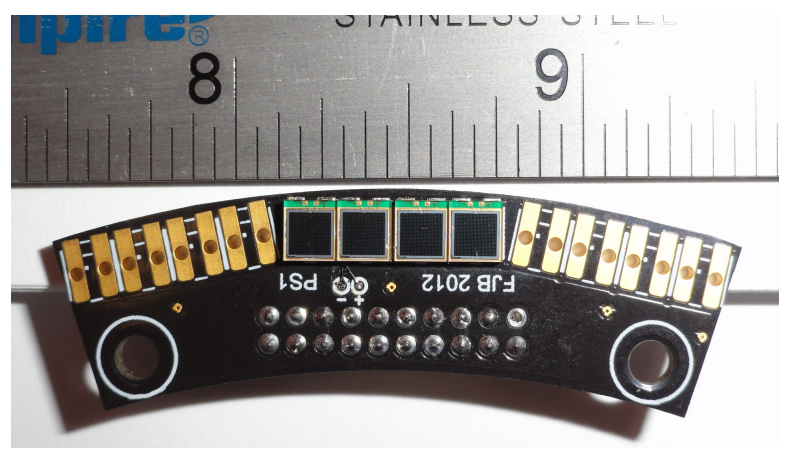

Figure 5: ST1 of Start Counter readout system. Only the central array is populated with SiPMs. Approximately $72 \%$ of the scintillator light is collected at the upstream end. The $\mathrm{ST}$ readout has $10 \mathrm{ST} 1$ units in total. The ruler shown above is in inches.

SiPMs has a thermocouple for temperature monitoring.

The second component "ST2", shown in Fig. 6. has three pre-amplifiers, three buffers, and three factor-five amplifiers. The output of each preamp is split; buffered for the analog-to-digital converter (ADC) output, and amplified for the time to digital converter (TDC) output. The ADC outputs are digitized by Jefferson Lab VXS 250 MHz Flash ADC modules 12]. The TDC outputs are input into Jefferson Lab leading edge discriminators, followed by a high resolution 32 channel Jefferson Lab VXS F1TDC V2 module[13]. Furthermore, the ST2 has three bias distribution channels with individual temperature compensation via thermistors. The ST2 is attached to the ST1 via a $90^{\circ}$ hermaphroditic connector.

The third component of the readout system, "ST3", provides the interface for the power and 


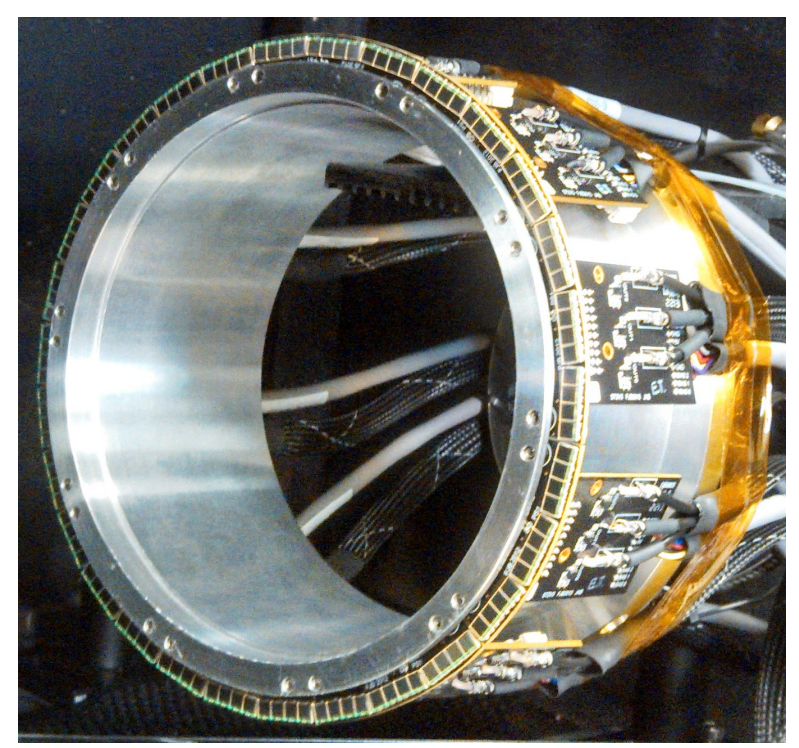

Figure 6: Fully assembled ST readout system. The ST2 unit is connected behind the ST1. The full readout system is comprised of $10 \mathrm{ST} 2$ units.

bias supplies. It also routes the ADC and TDC outputs as well as the thermocouple output. The ST3 is installed upstream of the Start Counter next to the beam pipe. A schematic of the ST readout electronics is illustrated in Fig. 7 .

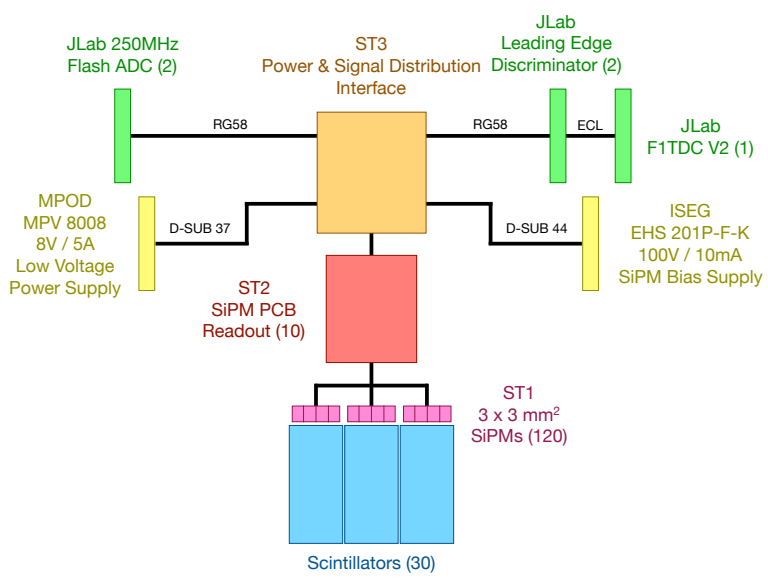

Figure 7: Start Counter readout electronics diagram. Numbers in parenthesis indicate the total for the system.

\section{Simulation}

In this section, Monte Carlo (MC) simulations of the performance and characteristics of machined scintillators are discussed. These studies were performed using the Geant4 tool-kit, which simulates the passage of particles through matter [14]. Comparisons are made with data observed in experiments conducted on the bench (Sec. 5.2) and with beam data (Sec. 7).

\subsection{Simulating a Simplified Model of the ST}

As discussed in Sec. 2.2, the ST paddle geometry has a nose section which tapers at the downstream end. This causes the light collection efficiency of hits in the nose section to increase as the hit position moves farther from the photo-detectors, contrary to the usual behavior of scintillator material.

A simple Geant4 simulation was conducted to investigate the light collection efficiency. The details of the simulation are discussed in Ref. 2]. Only the two trapezoidal regions of a machined scintillator paddle were considered: the wide straight section and the tapered nose section.

Ten thousand optical photons were generated at 16 different locations inside the medium of the scintillator. The photon energies ranged between 0.5 and $3.0 \mathrm{eV}[15]$ and were generated isotropically from points along a $3 \mathrm{~mm}$ path in the scintillator medium. This path is oriented orthogonal to the wide surface of the scintillator. The number of photons detected by the SiPM, denoted as "SiPM Hits", is shown in Figures 8 and 9 as a function of the source locations. For these studies, 100\% detection efficiency was assumed for the simulated SiPM. In the case of the nose section, the SiPM was placed at the wider upstream end of the simulated scintillator bar. The results for this simulation are presented in Fig. 8

The simulation shows that the tapering trapezoidal geometry of the nose section results in improved light collection as the source moves further away from the readout detector. There is an increase of $50 \%$ in light collection as the source is moved from the near end to the far end of the nose section. The quasi-rectangular straight section shows the typical loss off light yield as the source moves away from the photon detector.

\subsection{Simulating Machined Scintillator Geometry}

Further simulations were conducted to study the effects of the ST scintillator geometry and optical surface quality on light collection. The scintillator geometry was imported into Geant4 from a Vectorworks CAD drawing utilizing the CADMesh 


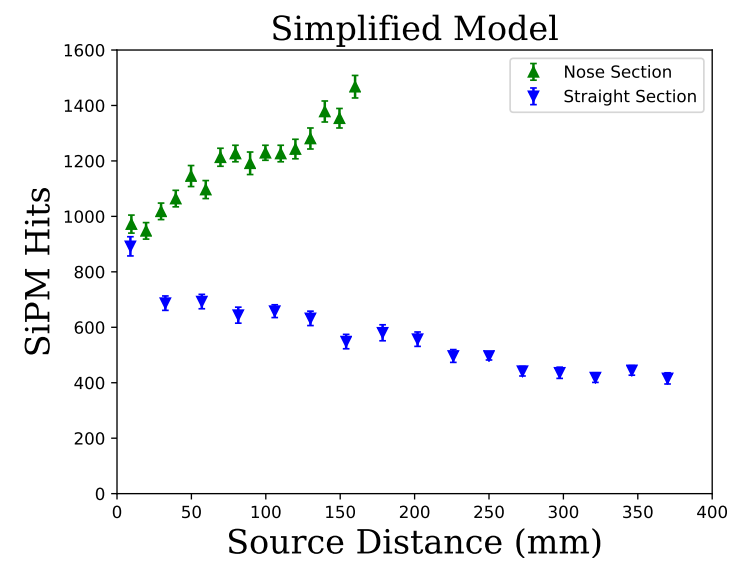

Figure 8: Simulation results for a simplified two section scenario. The total number of photons which were collected by the SiPM detector for each of the 16 source locations is plotted against the source distance from the photon detector.

utility [16]. The SiPM was modeled as a $12 \times 12 \times$ $10 \mathrm{~mm}^{3}$ volume with a $100 \mu \mathrm{m}$ air gap between it and the wide end of the straight section. The volume surrounding the scintillator was defined to be air. The scintillator material, SiPM photon detector, and optical photons were defined in an a manner identical to that discussed in Sec. 3.1.

To simulate the imperfections of scintillator surfaces, an optical surface "skin" was defined. The "skin" conformed to the POLISH and UNIFIED physics models 17] and was of the type "dielectricdielectric". Both the transmission efficiency and reflection parameters were implemented as free parameters in order to study their various effects on light transmission.

The POLISH model simulates a perfectly polished surface while the UNIFIED model defines the finish of the scintillator surface both of which are illustrated in Fig. 9 17]. The details of the UNIFIED model parameters are discussed in detail in References [2] \& 17].

As described in section 3.1, 10,000 optical photons were generated in the scintillator medium every $2.5 \mathrm{~cm}$ and the number of hits in the SiPM were recorded. For the POLISH model, only the transmission efficiency $\epsilon$ was varied. For the UNIFIED model, $\epsilon$ and the radiant intensity parameters were held constant while $\sigma_{\alpha}$, which characterizes the standard deviation of the surfaces micro-facet orientation, was varied. In both instances the attenuation length $\alpha$ was extracted in the straight section. The results are shown in Fig. 10.
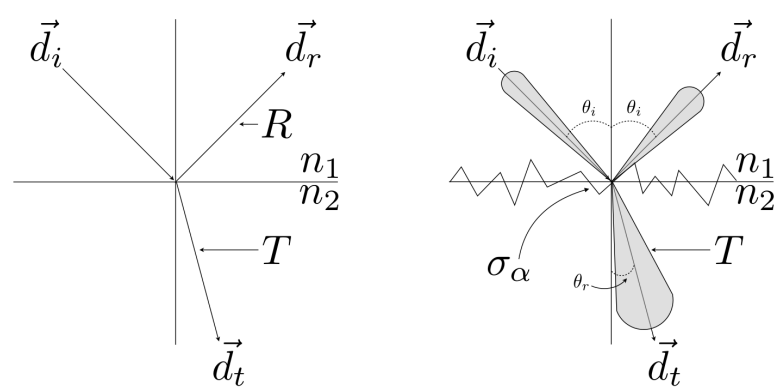

Figure 9: POLISH and UNIFIED models of scintillator surfaces. Left: Polar plot of the radiant intensity of the POLISH model. Right: Polar plot of the radiant intensity in the UNIFIED model [17. $\overrightarrow{d_{i}}, \overrightarrow{d_{r}}, \overrightarrow{d_{t}}$ are the incident, reflected, and refracted photon direction vectors respectively while $\overrightarrow{\sigma_{i}}$ and $\overrightarrow{\sigma_{r}}$ are the associated incident and reflected angles with respect to the average normals. $n_{1}$ and $n_{2}$ are the indices of refraction for the incident and transmission mediums respectively. $R$ is the probability of Fresnel reflection at the surface and the complementary probability of transmission is simply $T=1-R$.

For the POLISH model it is clear that if the transmission efficiency increases, i.e. the reflection efficiency decreases, the amount of light collected in the SiPM decreases as illustrated in Fig. 10. Similarly, as the number of micro-facet orientations increase, meaning a more coarsely ground surface, the amount of light collection in the SiPM also decreases. Moreover, good surface quality enhances the rise in light collection in the nose region.

\section{Misalignment Studies}

Here we discuss the relative alignment of a scintillator paddle with a SiPM detector and its effects on light collection and time resolution.

\subsection{Experimental Set-up}

The SiPM was mounted atop a Newport MTXYZ (MT) linear translation stage 18] with adjustment screws providing translations of $318 \mu \mathrm{m}$ per $360^{\circ}$ rotation. The SiPM collected light from a scintillator paddle at the upstream end of the straight section. A ${ }^{90} \mathrm{Sr}$ source and trigger photomultiplier tube (PMT) were fixed $24.5 \mathrm{~cm}$ downstream from the readout end. The response of the SiPM was recorded as a function of its relative position to the scintillator.

Utilizing a camera, the vertical and horizontal alignment of the SiPM relative to the scintillator was measured with $25 \mu \mathrm{m}$ accuracy. Further details of the experimental set-up are discussed in Ref. [2]. 

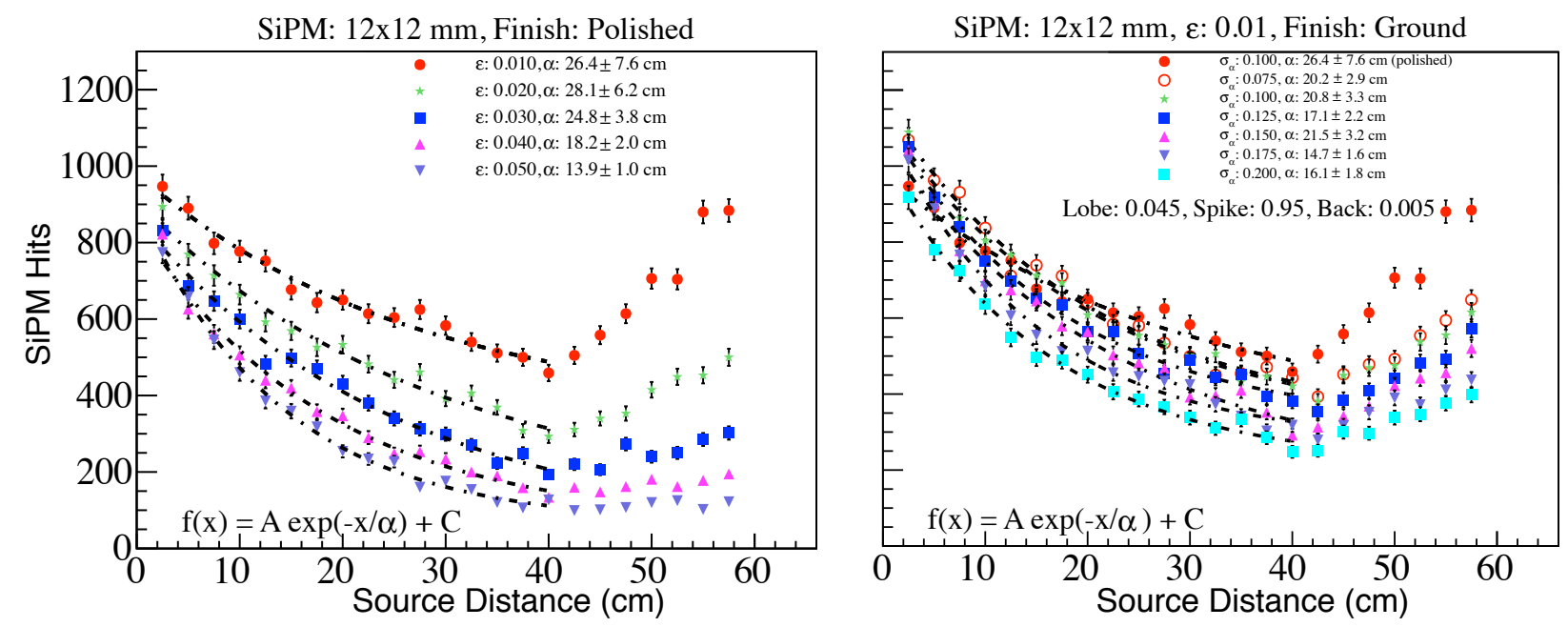

Figure 10: POLISH and UNIFIED model results. Shown is the number of hits recorded in the SiPM (vertical axis) versus the source distance (x-axis). Left: POLISH model varying the transmission efficiency $\epsilon$. Right: UNIFIED model varying the standard deviation of the surfaces micro-facet orientation $\sigma_{\alpha}$.

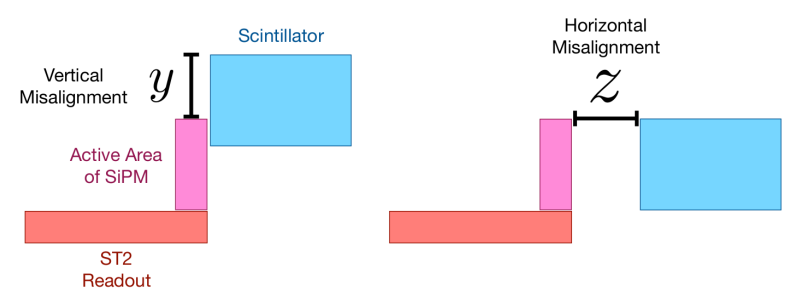

Figure 11: Optics setup for misalignment studies. Left: SiPM \& scintillator vertical misalignment. Right: SiPM \& scintillator horizontal misalignment.

\subsection{Vertical Alignment of SiPM and Scintillator}

The scintillator remained fixed while the SiPM was scanned across the upstream end of the scintillator (Fig. 11). During this scan, the horizontal alignment $(z)$ of the SiPM and scintillator was fixed at a distance of $100 \mu \mathrm{m}$ and was monitored closely. At $y=0$ the SiPM and scintillator are aligned vertically. The measurements and simulations are shown in Fig. 12. There is no significant variation of time resolution within a $\pm 300 \mu \mathrm{m}$ range of the optimal alignment.

A Geant4 simulation, done in a manner similar to that discussed in section 3.2, was utilized to study the effect of vertical misalignment. The photon collection statistics at various $y$-positions in simulation matched data taken on the bench. Ergo, the measured time resolution is dominated by photon collection statistics. Thus, we determined the simulated time resolutions empirically, by scaling light collection to the time resolutions measured on the

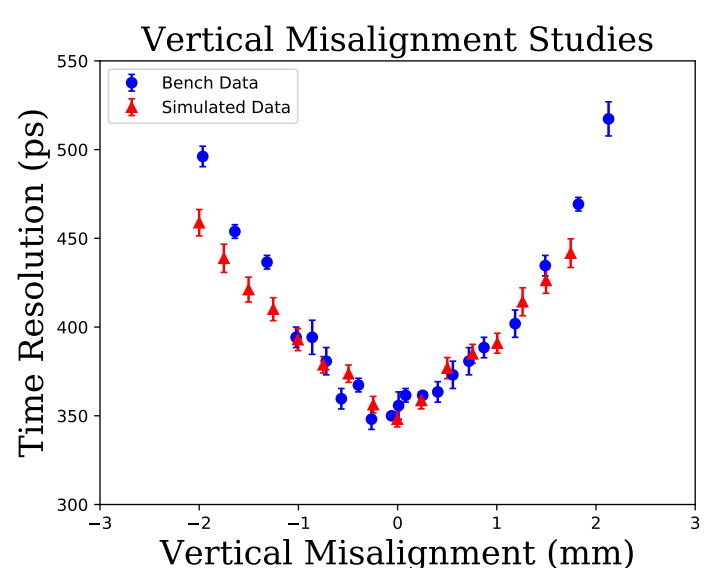

Figure 12: Vertical misalignment results. The minimum time resolution obtained was approximately 350 ps which was expected. Once the SiPM exceeded $y= \pm 3 \mathrm{~mm}$, no active area of the SiPM was directly coupled to the face of the scintillator.

bench. The acceptable range of vertical misalignment is approximately $\pm 250 \mu \mathrm{m}$.

\subsection{Horizontal Alignment of SiPM and Scintillator}

The effects of varying the horizontal alignment were also studied. While the horizontal alignment $(z)$ was varied, the vertical alignment $(y)$ was kept constant at the optimal location $(y=0)$, and was monitored both optically and manually with a micrometer. 
The SiPM was moved along the $z$-axis. We defined $z=0$ to be the position where the active area of the SiPM was flush against the face of the scintillator paddle. The results of this study are illustrated in Fig. 13. While the simulation underesti-

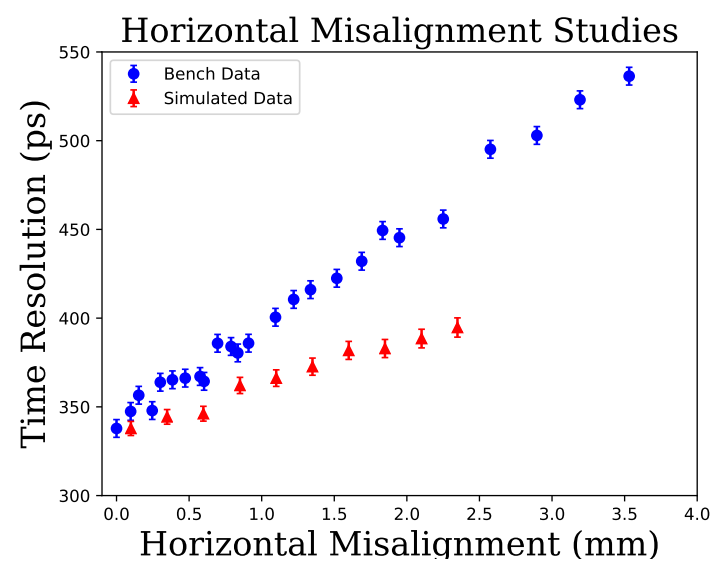

Figure 13: Horizontal misalignment results.

mates the degradation of resolution with increasing horizontal alignment, it is clear from the data that the optimal coupling range is $z<350 \mu \mathrm{m}$. Moreover, there is no significant degradation in time resolution for $z<600 \mu \mathrm{m}$.

\section{Fabrication}

The details of polishing and characterizing machined scintillators, as well as the construction of the Start Counter are discussed.

\subsection{Polishing Machined Scintillators}

While undergoing edge polishing at McNeil Enterprises, the machined scintillators incurred surface damage and were exposed to chemical contaminants known to harm scintillator surfaces. Polishing was required to restore adequate performance characteristics.

To polish the machined scintillator surfaces, Buehler Micropolish II deagglomerated $0.3 \mu \mathrm{m}$ alumina suspension was utilized [19]. The polishing suspension was diluted with a 5:1 ratio of de-ionized $\mathrm{H}_{2} \mathrm{O}$ to alumina and applied to a cold, wet $6^{\prime \prime} \times 0.5^{\prime \prime}$ Caswell Canton flannel buffing wheel 20] operated at speeds less than 1500 RPMs. The surfaces of the scintillators were carefully buffed until the large surface defects were removed. In order to eliminate small localized surface defects, hand polishing with a soft NOVUS premium Polish Mate microfilament cloth 21] and diluted polishing suspension was applied. These polishing procedures made the scintillators void of most visible scratches and surface defects.

The improved surface quality of the polished scintillators are shown in Fig. 14 where a scintillator paddle before and after polishing is shown. A red
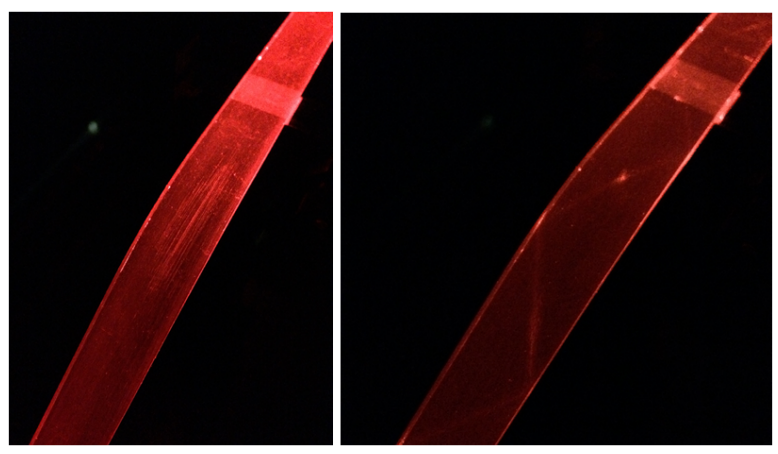

Figure 14: Effects of polishing scintillators. Left: non-diffuse laser incident on an edge, before polishing, at the upstream end of the straight section. Right: non-diffuse laser incident on the same edge, after polishing, at the upstream end of the straight section.

laser beam was shone into the scintillator medium from the upstream end aimed at one edge. The unpolished scintillator had such poor surface quality that the reflections of the laser in the bend region could not be resolved. However, the reflections in the polished scintillator can clearly be observed traversing the bend and nose region. On average, at the tip of the nose, the scintillators exhibited a $15 \%$ improvement in time resolution. Moreover, variation in performance from counter to counter was substantially reduced.

\subsection{Testing}

The polished scintillators were tested for light output and time resolution properties. A test stand (Fig. 15) was used to measure the response of machined scintillators at four locations in the straight section, three in the bend, and five in the nose.

The measurements were conducted with a collimated ${ }^{90} \mathrm{Sr}$ source oriented orthogonal to the wide flat surface of the scintillators. The ${ }^{90} \mathrm{Sr}$ source provides electrons ranging from $0.5-2.3 \mathrm{MeV}$ in energy 22] 23]. A trigger PMT was placed underneath the scintillator on the opposite side and provided the TDC start and ADC gate. A SiPM detector array identical the final ST assembly, collected 


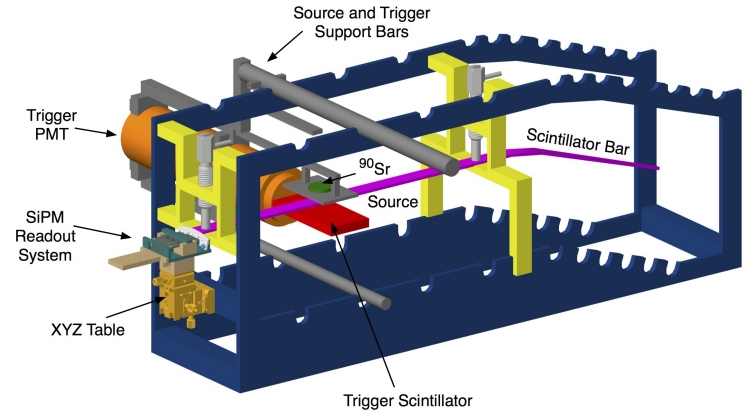

Figure 15: CAD Drawing of the scintillator test stand.

light from the scintillator being tested. The ADC and TDC data were analyzed to determine the light output and time resolution.

The 30 machined scintillator paddles that exhibited the best time resolution and light output properties from a set of 50 were selected for the final construction. These scintillators were then wrapped in $16.5 \mu \mathrm{m}$ thick reflective film (aluminum foil) and tested again. Their measured time resolutions are illustrated in Fig. 16. The phenomenon of increased

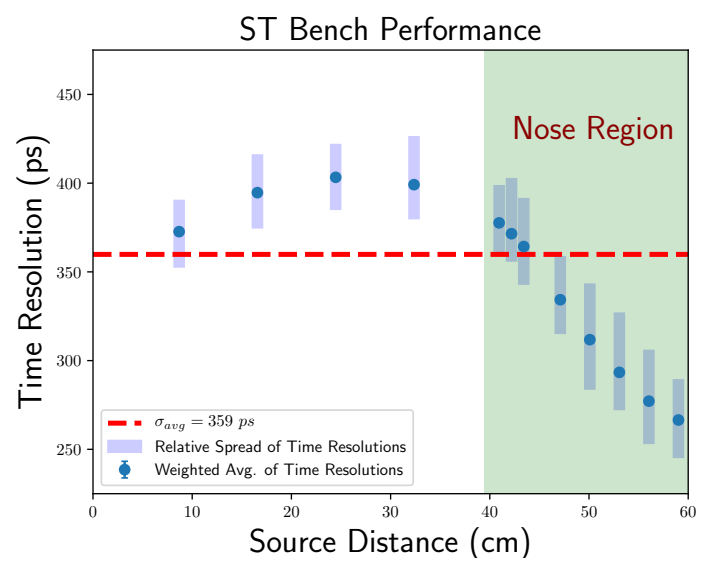

Figure 16: Weighted average of the time resolution of 30 scintillator paddles as a function of distance from the SiPM. The shaded vertical blue boxes indicate the relative spread of the time resolutions among the 30 paddles. The dashed line indicates the weighted average over the 12 data points.

light collection in the nose region is observed. The larger time resolution in the straight section is due to light which initially travels downstream is reflected from the nose.

\subsection{Assembly}

To build the ST an assembly jig (Fig. 17) was fabricated. The upstream support hub and Ro-

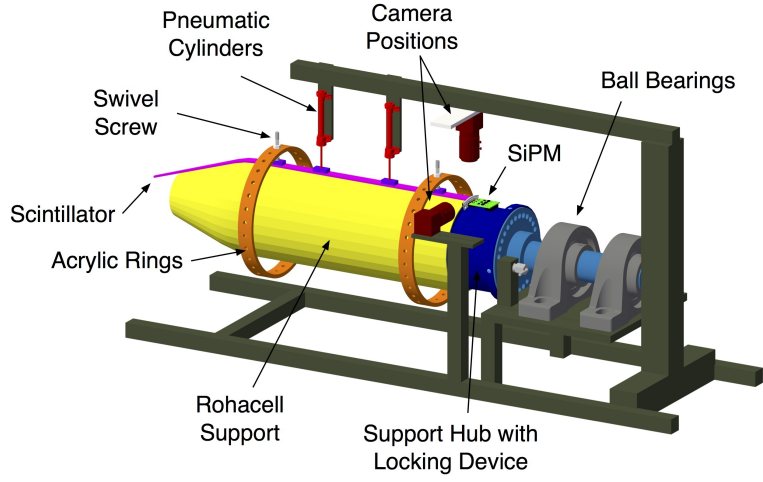

Figure 17: CAD drawing of the ST assembly jig.

hacell support structure were attached to a rotating bracket that moved in discretized $12^{\circ}$ increments. Two pneumatic cylinders with soft, semidense rubber feet were used to hold a single scintillator in place. Two free floating acrylic rings, with 30 tapped holes $12^{\circ}$ apart, housed $10^{\circ}$ swivel pad thumb screws fitted with silicone foam. The thumb screws held installed paddles in place.

A camera was used to measure and control the scintillator/SiPM vertical and horizontal alignments. Vertical alignment was achieved by using Kapton shims between the scintillator and the support structure. The horizontal alignment was configured to a distance less than $200 \mu \mathrm{m}$ between the scintillator and the SiPMs.

To secure paddles the to the Rohacell support structure the ST was wrapped around its circumference using self-adhesive transparent bundling wrap (0.8 mil thick, 6 in wide) at six locations along the length of the detector as seen in Fig. 18 . De-
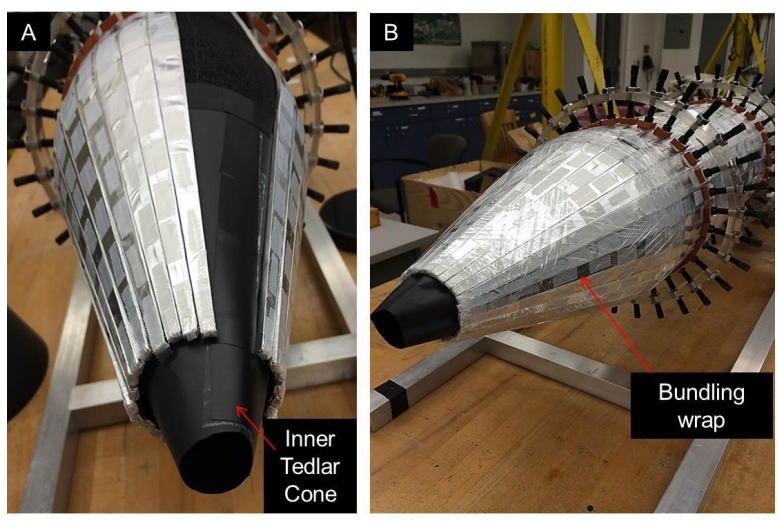

Figure 18: ST assembly before (A) and after (B) wrapping with bundling wrap 
tails regarding the assembly process are discussed in Ref. [2].

The fully assembled ST is mounted around the GlueX liquid $\mathrm{H}_{2}$ target as shown in Fig. 19.

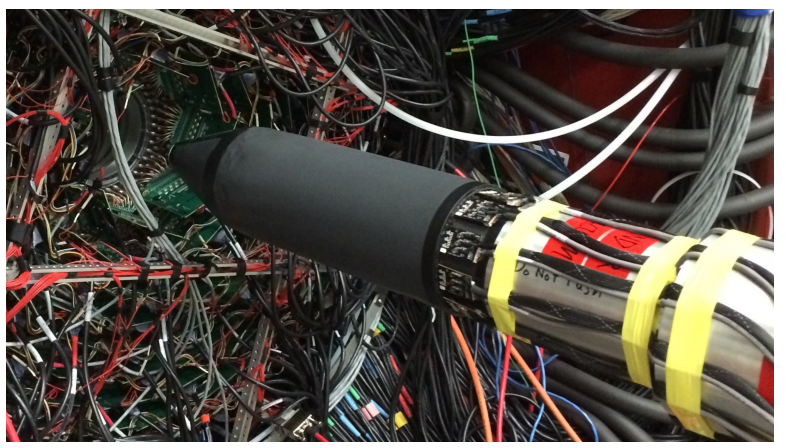

Figure 19: ST mounted the GLuEX target. The beam direction is from right to left and travels down the central axis of the ST. During operation the ST resides in the bore of the central tracking chamber, which is visible in the top left corner.

\section{Calibration}

The procedures to optimize the time resolution for particle identification (PID) and time of flight (TOF) are discussed here.

\subsection{Time-walk Correction}

To correct the TDC timing for variations due to pulse shape, we use the timing signal from the FADC250s. The latter uses a digital algorithm similar to a constant-fraction discriminator and therefore gives a time largely independent of pulse height 2] 24]. The TDC/FADC time difference is given by Eq. 1 where $i$ is the paddle number index.

$$
\delta t_{i}=t_{i}^{\mathrm{TDC}}-t_{i}^{\mathrm{FADC}}
$$

The FADC250's report the amplitude, integral, and time of the input analog signals 24. The amplitude was selected for the time-walk corrections because it is correlated better with the leading edge time of the pulse 2]. Figure 20 (left) shows a typical time-walk spectrum, i.e. $\delta t$ versus the pulse amplitude, for one paddle of the ST. This correlation can be described empirically by the function given by Eq. 2 25] where $a$ and $a_{i}^{\text {thresh }}$ are the pulse amplitude and discriminator threshold respectively, and $c 0_{i}, c 1_{i}, c 2_{i}$ are the fit parameters.

$$
f_{i}^{w}\left(a / a_{i}^{\text {thresh }}\right)=c 0_{i}+\frac{c 1_{i}}{\left(a / a_{i}^{\text {thresh }}\right)^{c 2_{i}}}
$$

The most probable value (MPV) of the pulse amplitude spectra was chosen as the reference point where the time-walk correction is defined to be zero. Fig. 20 (right) illustrates the effect on the time difference spectrum $(\delta t)$ as a result of the applied timewalk corrections.

\subsection{Propagation Time Corrections}

The time between the production of scintillation light in a ST scintillator paddle and detection by the SiPM depends on the hit location along the paddle and is discussed below.

The EJ-200 scintillator material has a refractive index of 1.58 [5] and the corresponding speed of light in that medium is $19 \mathrm{~cm} / \mathrm{ns}$. The observed effective velocity is slower. Correcting for this light propagation in the scintillator is necessary since the ST paddles are $60 \mathrm{~cm}$ long. Studies showed that the effective velocity of light depends on the region along the paddle where the hit occurred. The propagation time corrections were conducted with welldefined reconstructed charge particle tracks. Further details regarding the event and track selection are found in Ref. [2].

The propagation time $T_{\text {prop }}^{\mathrm{ST}}$ is determined by Eq. 3 where $T_{\text {hit }}^{\mathrm{ST}}$ is the time-walk corrected hit time, $T_{\text {flight }}^{\mathrm{ST}}$ is the flight time from the track vertex to the ST intersection point, and $T_{\text {vertex }}^{\mathrm{BB}}$ is the track vertex time.

$$
T_{\mathrm{prop}}^{\mathrm{ST}}=T_{\mathrm{hit}}^{\mathrm{ST}}-T_{\text {flight }}^{\mathrm{ST}}-T_{\mathrm{vertex}}^{\mathrm{BB}}
$$

The $z$-coordinate of the track's intersection point with the ST $\left(z_{\text {hit }}^{\mathrm{ST}}\right)$ are determined by the detector geometry as well as the distance $d_{\text {hit }}^{\mathrm{ST}}$ of this intersection point and the SiPM.

The propagation times were determined in three distinct regions corresponding to the three geometrical sections of the ST: the straight, bend, and nose regions. The propagation times in these regions were fit with a linear function given by Eq. 4 where $j$ indicates which region in the $i^{\text {th }}$ paddle is being fit and $A$ and $B$ are fit parameters.

$$
f_{j}^{i}(z)=A_{j}^{i}+B_{j}^{i} \cdot z
$$

Figure 21 (left) illustrates the correlation between the propagation time and the distance from the SiPM with $T_{\text {prop }}^{\mathrm{ST}}=0.0 \mathrm{~ns}$ when $d_{\text {hit }}^{\mathrm{ST}}=0.0 \mathrm{~cm}$. Figure 21 (right) illustrates the corrected time. 

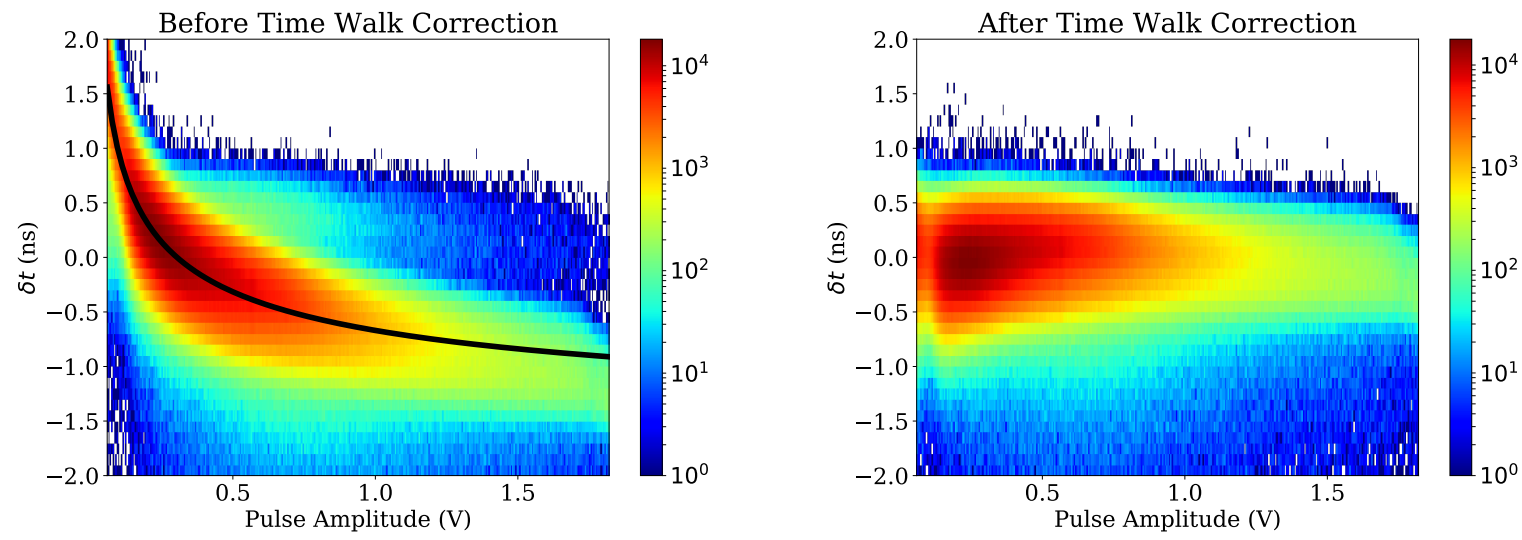

Figure 20: Left: Single paddle time-walk spectrum; the line shown is the fitted function used to determine the correction factors. Right: after time-walk correction. Plotted on the vertical axis is $\delta t$ and on the horizontal axis is the corresponding pedestal subtracted pulse amplitude spectrum.
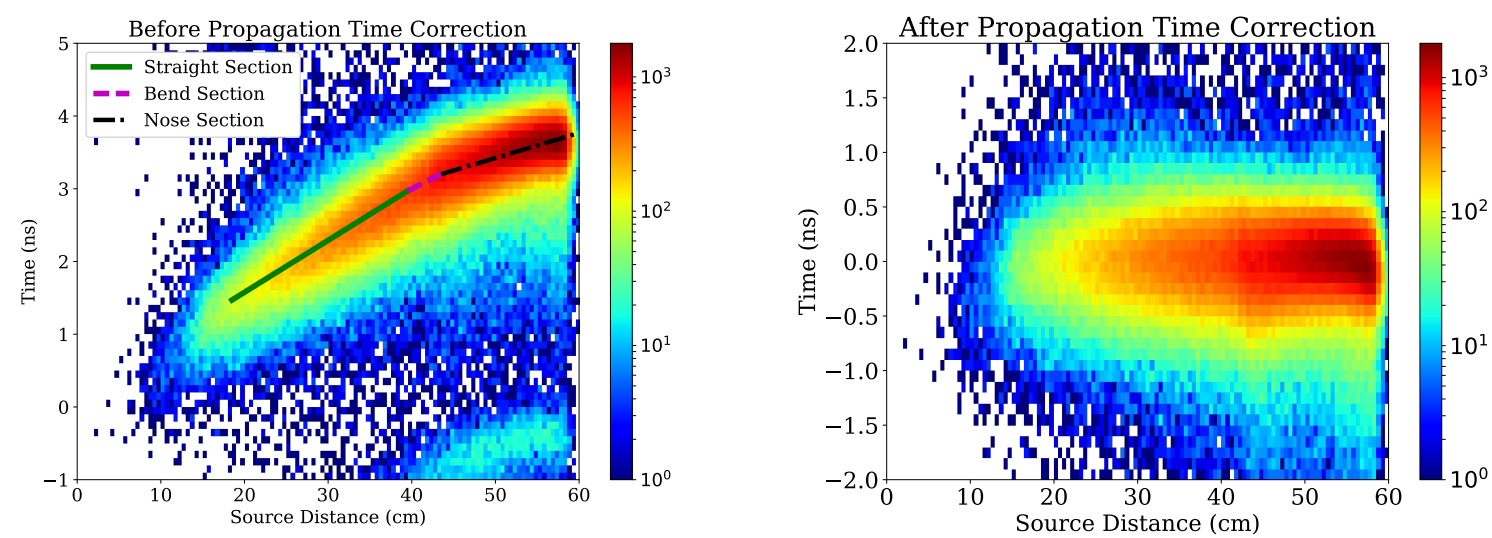

Figure 21: Left: Single paddle propagation time correlation. $T_{\mathrm{prop}}^{\mathrm{ST}}$ is plotted on the vertical axis and $d_{\text {hit }}^{\mathrm{ST}}$ is plotted along the horizontal axis. There is a clear correlation between the time when optical photons are detected by the SiPM and the location of the scintillation light along the length of the paddle. Right: Single paddle propagation time after correction.

\subsection{Attenuation Corrections}

To measure attenuation in the scintillators, charged tracks were selected in a manner similar to that discussed in Sec. 6.2. The uncorrected energy deposition $\left(d E_{M}\right)$ per unit length $(d x)$ versus the track momentum $(p)$ for tracks intersecting to the ST are shown in Fig. 25. It is clear that no reliable PID can occur for tracks with $p>0.6 \mathrm{GeV} / \mathrm{c}$ without further corrections.

The pulse integral (PI) data, normalized to the path length $d x$ of the track in the scintillator medium, were binned in $3.5 \mathrm{~cm} z_{\text {hit }}^{\mathrm{ST}}$ bins along the length of the paddle. The MPV of the PI was extracted utilizing an empirical function given by Eq. 5 where $p_{0}, p_{1}, p_{2}$ are the fit parameters.

$$
f(z)=p_{0} e^{\left(-p_{1}\left(z-p_{2}\right)\right)} \times\left(1+\tanh \left(p_{1}\left(z-p_{2}\right)\right)\right)
$$

A fit to the data in a single $3.5 \mathrm{~cm} z_{\text {hit }}^{\mathrm{ST}}$ bin is illustrated in Fig. 22. The MPV was extracted analytically and then plotted against the average value for each $z_{\text {hit }}^{\mathrm{ST}}$ bin as shown in Fig. 23 .

In order to characterize the photon attenuation, the straight and nose regions were treated independently. The piecewise continuous function given by Eq. 6 was selected to fit the data where the intersection $Z_{b}^{i}$ (or correction boundary) of the two 


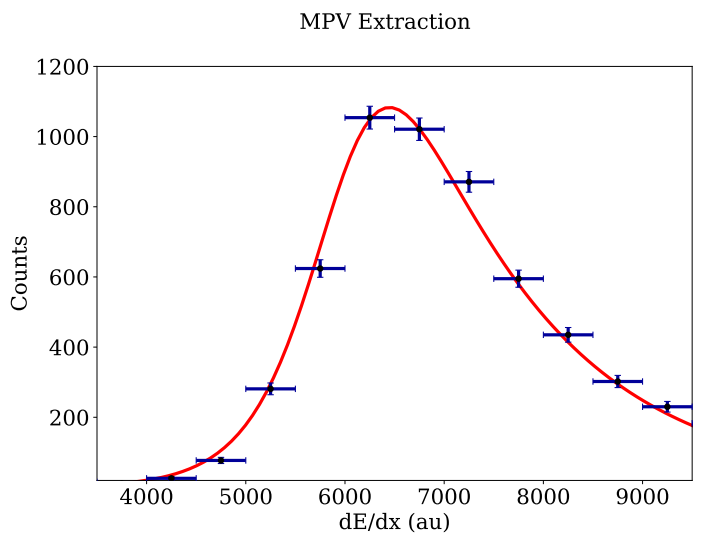

Figure 22: Pulse integral integral data normalized to the the track length in the scintillator medium for a single $3.5 \mathrm{~cm}$ bin along the paddle length.

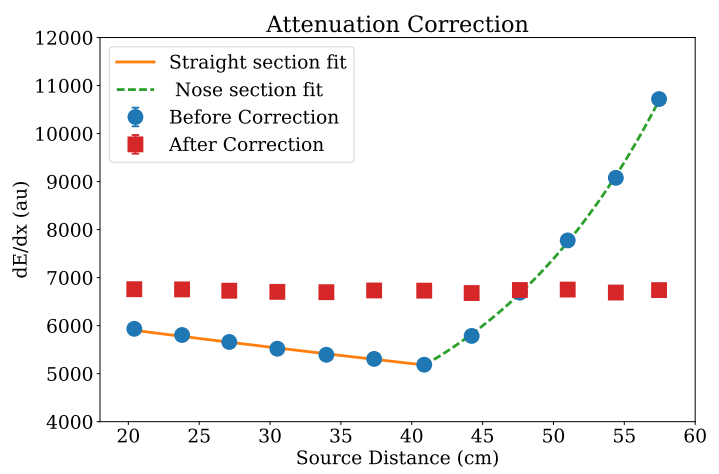

Figure 23: Fits to the attenuation data.

exponential fit functions was fixed and is shown in Fig. 23.

$$
f_{c}^{i}(z)= \begin{cases}A_{S}^{i} e^{-B_{S}^{i} \cdot z} & z \leq Z_{b}^{i} \mathrm{~cm} \\ A_{N}^{i} e^{B_{N}^{i} \cdot z}+C_{N}^{i} & z>Z_{b}^{i} \mathrm{~cm}\end{cases}
$$

In Eq. 6, the subscripts $S$ and $N$ denote the straight and nose sections respectively while $A^{i}, B^{i}$, and $C^{i}$ are the fit parameters for the $i^{t h}$ paddle.

An attenuation correction factor $R^{i}(z)$ is applied to the deposited energy measurement per unit track-length $\left(d E_{M} / d x\right)$ to give the corrected energy deposition per unit track length $\left(d E_{C}^{i}(z) / d x\right)$ for paddle $i$ and is given by Eq. 7 where the subscripts $C$ and $M$ are the corrected and measured quantities respectively.

$$
\frac{d E_{C}^{i}(z)}{d x}=\frac{d E_{M}}{d x} \cdot R^{i}(z)=\frac{d E_{M}}{d x} \cdot \frac{f_{c}^{i}(0)}{f_{c}^{i}(z)}
$$

After attenuation corrections are applied, particle separation is greatly improved. This will be discussed further in Sec. 7 .

\section{Performance}

The increase in light output as a function of hit position along the ST detector during nominal GLUEX beam conditions is illustrated in Fig. 24. This is advantageous because the majority of the

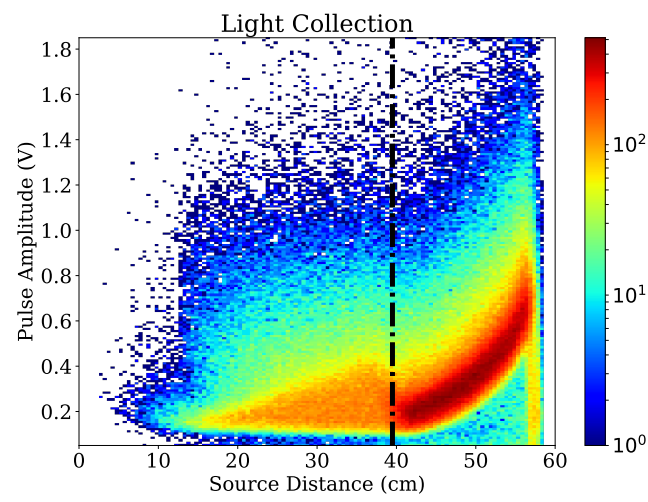

Figure 24: Typical FADC250 pulse amplitude spectrum versus the $z$-component of charged tracks intersecting the ST for an individual ST sector. The vertical line indicates the start of the tapered nose section.

charged tracks produced intersect the ST in the forward region.

With the attenuation corrections discussed in Sec. 6.3 applied to the data, the PID capabilities of the ST were improved. Figure 25 illustrates the PID capability of charged tracks intersecting the ST. Protons can be separated from other hadrons with momenta up to $0.9 \mathrm{GeV} / \mathrm{c}$ which is a factor 1.5 improvement relative to the uncalibrated data. The PID capabilities of the ST extend the identification of low momentum protons that do propagate through the central drift chambers.

The ST was used to determine the time of the interaction of a beam photon with the $\mathrm{LH}_{2}$ target after the time-walk and propagation time corrections discussed in Sec. 6.1 and 6.2 were applied. The interaction time can be determined independently from a timing signal originating from the accelerators RF system, the latter with very high precision. The time difference between the ST time and the machine RF time is shown in Fig. 26. The accelerator can be run in mode where the time separation between beam bunches is 2 ns, a separation indicated in the figure. One application of the ST is to 

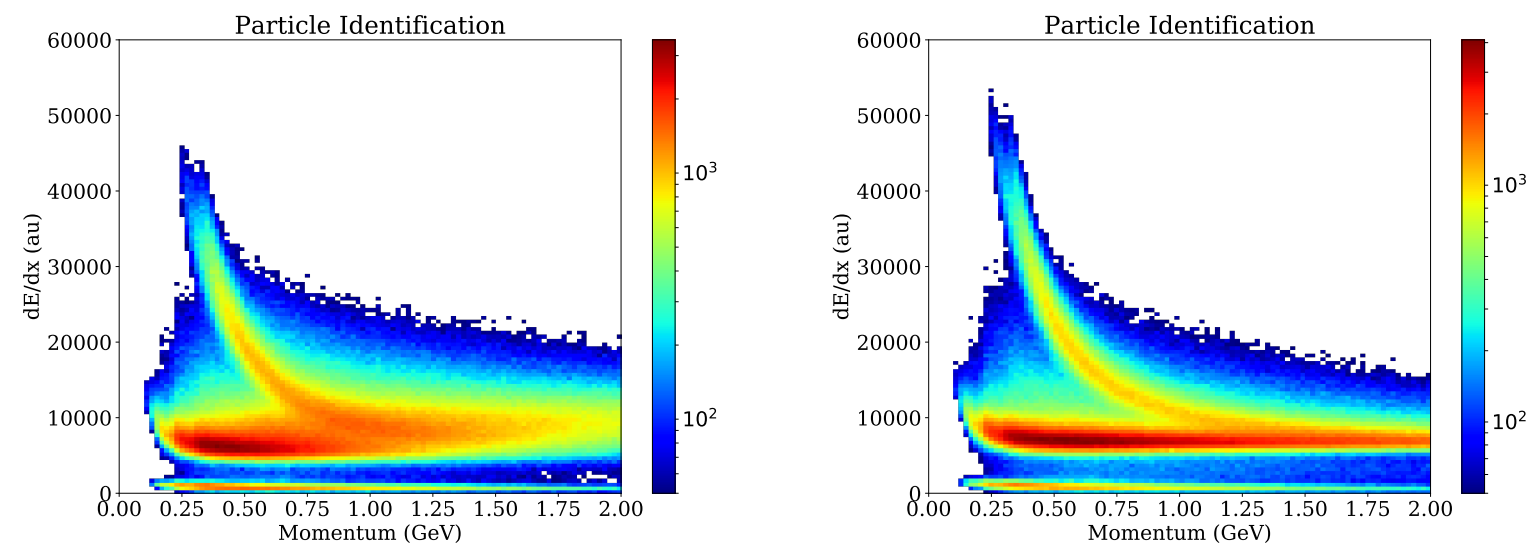

Figure 25: Left: Typical uncorrected $d E / d x$ vs. $p$ distribution. Right: Corrected $d E / d x$ vs. $p$ distribution. The "banana band" corresponds to protons while the horizontal band corresponds to electrons, pions, and kaons. It is clear that after the corrections have been applied, pion/proton separation is achievable for tracks with $p<0.9 \mathrm{GeV} / \mathrm{c}$.

distinguish particles from different RF buckets on the basis of timing.

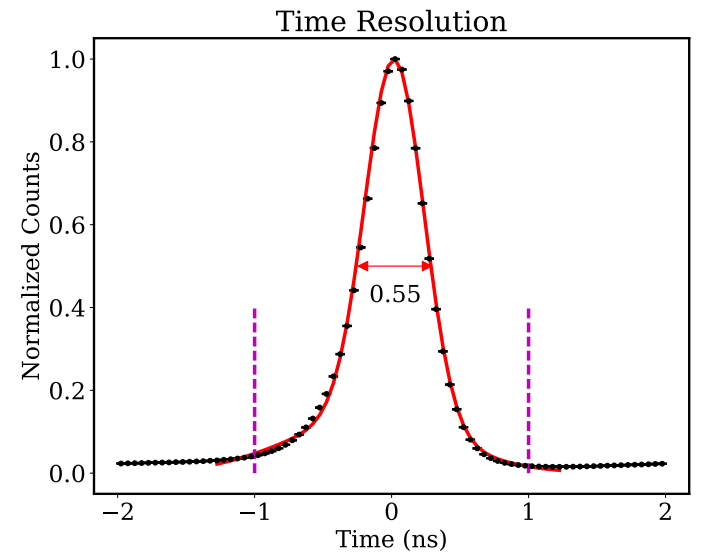

Figure 26: Time resolution for one paddle with its full width half maximum value indicated in $\mathrm{ns}$. The $\mathrm{x}$-axis is the time difference between $T_{\text {vertex }}^{\mathrm{ST}}$ and $T_{\mathrm{vertex}}^{\mathrm{BB}}$. The vertical lines indicate the cuts used to identify a $500 \mathrm{MHz}$ beam bunch.

The measured distribution is fit with the sum of two Gaussians and the full width at half maximum (FWHM) of the resulting curve is calculated. Also, the fraction of the area of the curve within $\pm 1 \mathrm{~ns}$ of zero is calculated. Fits were carried out for the three individual geometrical regions and for all 30 paddles. The results are shown in Table 2.

The ST exhibited uniformity in time resolution among all sectors of the ST. The high overall event fraction and good time resolution in the data for all

\begin{tabular}{ccccc}
\hline Section & All & Straight & Bend & Nose \\
\hline FWHM & $550 \mathrm{ps}$ & $690 \mathrm{ps}$ & $700 \mathrm{ps}$ & $450 \mathrm{ps}$ \\
Fraction & $93 \%$ & $92 \%$ & $91 \%$ & $94 \%$ \\
\hline
\end{tabular}

Table 2: Average time resolutions (FWHM) and event fractions within a \pm 1 ns window for all 30 ST sectors by independent geometrical regions.

sections combined is due to the majority of events intersecting the ST in the nose section. It is clear from Table 2 that measurements made with beam data also exhibit improved light collection, and thus improved time resolution, in the nose region.

Approximately four years of operation have elapsed since the paddles were first tested on the bench at Florida International University (FIU). Prior experience with scintillators indicates that degradation in time resolution as a result of mishandling will be visible in a matter of weeks. No degradation in time resolution has been observed and the ST is still performing well below design resolution.

\section{Conclusion}

The GlueX Start Counter was designed and constructed at Florida International University for use in Hall D at TJNAF. It provides separation of the $500 \mathrm{MHz}$ photon beam bunch structure delivered by the CEBAF to within $94 \%$ accuracy. It is the first "start counter" detector to utilize magnetic field 
insensitive SiPMs as the readout system. Despite the many design and manufacturing complications, the ST has proven to have performed well beyond the design resolution of 825 ps (FWHM) with an average measured resolution of 550 ps (FWHM). Furthermore, the capabilities of the ST make it a viable candidate to assist in particle identification.

The unique geometry of the ST nose section has illustrated the advantage of tapering trapezoidal geometry in thin scintillators. Through simulation, tests on the bench, and analysis of data obtained with beam, it has been definitively demonstrated that this geometry results in a phenomenon in which the amount of light detected increases as the scintillation source moves further downstream from the readout detector.

Since its installation in Hall D during the Fall 2014 commissioning run, the ST has shown no measurable signs of deterioration in performance. This suggests that the ST scintillators are void of crazing and will most likely be able to meet and exceed the design performance well beyond the scheduled run periods associated with the GLUEX experiment.

It is planned to incorporate the ST into the level 1 trigger of the GLUEX experiment for high luminosity running when there will be $5 \times 10^{7} \gamma / s$ in the coherent peak. Preliminary studies suggest that while operating at rates in excess of $300 \mathrm{kHz}$ per paddle, the ST exhibits a high efficiency ( $>95 \%$ ). Thus, in combination with the calorimeters the ST has the potential to provide good suppression of electromagnetic background via incorporation into the level 1 trigger of the experiment. Furthermore, the ST's high degree of segmentation has demonstrated suppression of various background contributions associated with complex topologies while simultaneously providing precision timing information for reconstructed charged particles in GLUEX.

\section{Acknowledgments}

The authors would like to graciously thank the plethora of Jefferson Lab staff members in both the Engineering division and Hall-D. Their numerous and invaluable contributions allowed for the Start Counter project to come to fruition. The authors would also like to extend their gratitude to the entire GLuEX Collaboration who provided fruitful ideas and advice throughout the many stages of the project. Work at Florida International University was supported in part by the Department of Energy under contracts DE-FG02-99ER41065 and DE-SC00-13620. Furthermore, this material is based upon work supported by the U.S. Department of Energy, Office of Science, Office of Nuclear Physics under Contract No. DE-AC0506OR23177. 


\section{References}

[1] H. Al Ghoul, et al., First results from the gluex experiment AIP Conference Proceedings 1735 (1) (2016) 020001. arXiv:https://aip.scitation.org/doi/pdf/ 10.1063/1.4949369 doi:10.1063/1.4949369 URL https://aip.scitation.org/doi/abs/10.1063/ 1.4949369

[2] E. Pooser, The GlueX start counter and beam asymmetry $\Sigma$ in single $\pi^{0}$ photoproduction, Ph.D. thesis, Florida International University, http:// digitalcommons.fiu.edu/etd/2450/ (2016).

[3] A. Somov, Performance of the level-1 trigger at high luminosity, talk Given at the January 19, 2017 GlueX Collaboration meeting available at https://halldweb.jlab.org/DocDB/0031/003193/ 004/high_rate_status.pdf (2017).

[4] http://www.eljentechnology.com/

[5] General Purpose Plastic Scintillator EJ-200, EJ204, EJ-208, EJ-212, technical note available at http://www.eljentechnology.com/images/products/ data_sheets/EJ-200_EJ-204_EJ-208_EJ-212.pdf

[6] http://www.mcnealplasticmachining.com/

[7] http://www.rohacell.com

[8] http://www.dupont.com/products-and-services/ membranes-films/pvf-films.html

[9] http://www.hamamatsu.com/us/en/index.html

[10] F. Barbosa, Time characteristics of silicon photomultipliers used in the gluex experiment, in: 2015 IEEE Nuclear Science Symposium and Medical Imaging Conference (NSS/MIC), 2015, pp. 1-4, available at http: //ieeexplore.ieee.org/document/7581740/

[11] Multi-Pixel Photon Counter, technical note available at https://halldweb1.jlab.org/wiki/images/4/ 49/S10362-33_series_kapd1023e05.pdf

[12] F. B. et al., A VME64x, 16 Channel, Pipelined 250 MSPS Flash ADC With Switched Serial (VXS) Extension, Jefferson Lab, technical note GlueX-doc-1022 available at https://halldweb.jlab.org/doc-public/ DocDB/ShowDocument?docid=1022 (April 2008).

[13] F. B. et al., The Jefferson Lab High Resolution Time-toDigital Converter (TDC), Jefferson Lab, technical note GlueX-doc-1021 available at https://halldweb.jlab. org/doc-public/DocDB/ShowDocument?docid=1021 (April 2008).

[14] https://geant4.web.cern.ch/geant4/

[15] K. S. Krane, Introductory Nuclear Physics, John Wiley \& Sons, 1988, Ch. Detecting Nuclear Radiations, pp. 201-202.

[16] C. M. Poole, A cad interface for geant4, paper submitted to Cornell Archive (arXiv) May 5, 2011 available at http://arxiv.org/pdf/1105.0963.pdf (2011).

[17] A. L. . C. Moison, A more physical approach to model the surface treatment of scintillation counters and its implementation into detect, paper presented at IEEE, Nuclear Science Symposium; Anaheim, November 2-4 available at http://geant4.slac.stanford. edu/UsersWorkshop/PDF/Peter/moisan.pdf (1996).

[18] https://www.newport.com/p/MT-XYZ.

[19] https://shop.buehler.com/consumables/ grinding-polishing/polishing-suspensions/ alumina-suspensions

[20] http://www.caswellplating.com/

buffing-polishing/buffing-wheels/

canton-flannel-wheels.html\#
[21] https://www.novuspolish.com/polish_mates.html

[22] ${ }^{90} \mathrm{Sr}$ Decay Radiation Data, national Nuclear Data Center, Nuclear Structure and Decay Database (NuDat) available at http://www.nndc.bnl.gov/ nudat $2 /$ decaysearchdirect. jsp?nuc $=90$ SR\&unc $=$ nds

[23] ${ }^{90} Y$ Decay Radiation Data, national Nuclear Data Center, Nuclear Structure and Decay Database (NuDat) available at http://www.nndc.bnl.gov/ nudat $2 /$ decaysearchdirect. jsp?nuc $=90$ Y \&unc $=$ nds

[24] H. Dong, Description and Instructions for the Firmware of Processing FPGA of the ADC250 Boards Version 0x0C0D, fADC250 Manual Available at https://coda.jlab.org/drupal/system/files/pdfs/ HardwareManual/fADC250/FADC250_Processing_FPGA_ Firmware_ver_0x0COD_Description_Instructions . pdf (February 2017).

[25] E. Smith, Low-level calibration constants for BCAL, technical note GlueX-doc-2618-v10, available at https://halldweb.jlab.org/DocDB/0026/002618/ 011/bcal_constants.pdf (December 2014). 